\title{
NORMAL DIVISION ALGEBRAS OVER A MODULAR FIELD*
}

\author{
BY \\ A. ADRIAN ALBERT
}

1. Introduction. Let $\phi(\omega)=0$ have coefficients in a modular field $F$ of characteristic $p$ and be irreducible in $F$. Then $\phi(\omega)=0$ and the field $F(x)$ generated by any one of its roots $x$ are called separable or inseparable according as $\dot{\phi}(\omega)=0$ has not or has multiple roots. It is well known $\dagger$ that if $\phi(\omega)=0$ is inseparable, then

$$
\phi(\omega) \equiv \sum_{i} \alpha_{i} \omega^{p i}
$$

$\left(\alpha_{i}\right.$ in $\left.F\right)$,

and that there exist inseparable extensions $F(x)$ of $F$ if and only if some quantity $\alpha$ of $F$ is not the pth power of any quantity of $F$.

An infinite field $F$ is called perfect if either $F$ is non-modular or every quantity of $F$ has the form $\beta^{p}$ where $p$ is the characteristic of $F$ and $\beta$ is in $F$. In any consideration of normal division algebras $D$ over $F$ the property that $F$ is perfect is used only when we consider quantities of $D$ and the minimum equations of these quantities. But if the degree $n$ of $D$ is not divisible by the characteristic $p$ of $F$, then the assumption that $F$ is perfect evidently has no value and is a needless extremely strong restriction on $F$.

In most of the papers on the structure of normal division algebras written recently in Germany $\ddagger$, the assumption has been that $F$ is perfect. But I shall prove here that if $F$ is perfect of characteristic $p$, then $n$ is not divisible by $p$. Hence it is now necessary to consider algebras of degree $p^{e}$ over $F$ of characteristic $p$, where $F$ is not perfect.

I shall give here a brief discussion of the validity of the major results on algebras over non-modular fields when $F$ is assumed to be merely any infinite field. Moreover, I shall determine all normal division algebras of degree two over $F$ of characteristic two, of degree three over $F$ of characteristic three. $\S$

2. The existence of a maximal separable sub-field of $A$. Let $A$ be any normal division algebra of degree $n$ over any field $F$, and let

$$
u_{1}, \cdots, u_{m}
$$$$
\left(m=n^{2}\right)
$$

* Presented to the Society, December 1, 1933; received by the editors November 22, 1933.

$\dagger$ Cf. B. L. van der Waerden's Moderne Algebra for the theory of modular fields.

$\ddagger$ In particular the papers by $R$. Brauer.

$\$$ I have also completed a determination of all normal division algebras of degree four over $F$ of characteristic two and have offered this more complicated determination for publication in the American Journal of Mathematics. 
be a basis of $F$. Then it is known* that if $K$ is an algebraically closed extension of $F$, the algebra $A_{K}$ over $K$ is a total matric algebra $M$. Let

$$
e_{\alpha \beta}=v_{j}=\sum_{i=1}^{m} \mu_{j i} u_{i}, \quad u_{i}=\sum_{i=1}^{m} \lambda_{i j} v_{j} \quad(i, j=1, \cdots, m) .
$$

where $\alpha, \beta=1, \cdots, n$ and $j=(\alpha-1) n+\beta$. The quantities $\lambda_{i j}, \mu_{j i}$ are then in $K$ and $e_{\alpha \beta}$ corresponds to an $n$-rowed matrix with unity in the $\alpha$ th row and $\beta$ th column and zero elsewhere.

The rank equation of $A$ is the minimum equation of the quantity $x=\sum_{i-1}^{m} \xi_{i} u_{i}$ where the $\xi_{i}$ are independent variables. Then it is known that we have the result $\dagger$

THEOREM 1. The rank equation of $A$ is the characteristic equation of the matrix

$$
\left\|\zeta_{\alpha \beta}\right\| \quad(\alpha, \beta=1, \cdots, n)
$$

where

$$
\zeta_{\alpha \beta}=\sum_{i=1}^{m} \mu_{j i} \xi_{i}, \quad j=(\alpha-1) n+\beta .
$$

This equation has coefficients in $L=F\left(\xi_{1}, \cdots, \xi_{m}\right)$ and is irreducible in $L$.

E. Noether and G. Köthe have given proofs $\ddagger$ of

THEOREM 2. Algebra $A$ of degree $n$ over an infinite field $F$ has separable subfields $F(x)$ of degree $n$.

Their proofs are not at all elementary while my very much earlier simpler proof $\S$ for the case where $F$ is non-modular holds and uses only Theorem 1. We may in fact prove

THEOREM 3. The sub-fields $F(x)$ of Theorem 2 may be so chosen that $x$ satisfies

$$
\omega^{n}+\lambda_{1} \omega^{n-1}+\cdots+\lambda_{n}=0 \quad\left(\lambda_{1} \neq 0, \lambda_{i} \text { in } F\right) .
$$

For the rank equation $R\left(\omega ; \xi_{1}, \cdots, \xi_{m}\right)$ is satisfied by any matrix (3) when the corresponding values of $\xi_{1}, \cdots, \xi_{m}$ are given. Let $\beta_{1}, \cdots, \beta_{n}$ be $n$ quantities of the infinite field $F$ so chosen that $\beta_{1}, \cdots, \beta_{n-1}$ are distinct

* Cf. van der Waerden's Algebra, II, p. 176.

$\dagger$ For proof of Theorem 1, see L. E. Dickson's Algebren und ihre Zahlentheorie, pp. 259-262. Dickson's proof uses only (2) and is an immediate consequence of his Theorem 5 without the argument of the unnecessary section 132 .

‡ Journal für Mathematik, vol. 166 (1932), pp. 182-184, for Köthe's proof, and Mathematische Zeitschrift, vol. 37 (1933), pp. 514-541, p. 535 for Noether's proof.

\& Bulletin of the American Mathematical Society, vol. 36 (1930), pp. 649-650. 
and $\beta_{n} \neq \beta_{i},-\left(\beta_{1}+\cdots+\beta_{n-1}\right)$ for $i=1, \cdots, n-1$. Then we solve (4) for the $\xi_{i}$ and have proved the existence of $\xi_{i 0}$ in $K$ for which $R\left(\omega ; \xi_{10}, \cdots, \xi_{m 0}\right)$ $=0$ has distinct roots and the coefficient $\lambda_{1}\left(\xi_{10}, \cdots, \xi_{m 0}\right)$ of $\omega^{n-1}$ is not zero. Let $D\left(\xi_{1}, \cdots, \xi_{n}\right)$ be the discriminant of $R\left(\omega ; \xi_{1}, \cdots, \xi_{m}\right)$. Then

$$
D\left(\xi_{10}, \cdots, \xi_{m 0}\right) \lambda\left(\xi_{10}, \cdots, \xi_{m 0}\right) \neq 0,
$$

so that $D\left(\xi_{1}, \cdots, \xi_{m}\right) \cdot \lambda\left(\xi_{1}, \cdots, \xi_{m}\right) \not \equiv 0$. But then there exist values $\xi_{i 1}$ of $\xi_{1}, \cdots, \xi_{m}$ in $F$ such that $D\left(\xi_{11}, \cdots, \xi_{m 1}\right) \cdot \lambda\left(\xi_{11}, \cdots, \xi_{m 1}\right) \neq 0$ and hence such that the rank equation of $A$ for $x=\sum \xi_{i 1} u_{i}$ has distinct roots and coefficient of $\omega^{n-1}$ not zero.

The characteristic equation of the corresponding matrix (3) is an exact power of the minimum equation of $x$ since $x$ in the division algebra $A$ has irreducible minimum equation. Since the characteristic equation has been shown to have distinct roots, it is the minimum equation of $x$ and we have proved Theorems 2, 3 .

3. Known theorems. In this section we shall state certain well known theorems on algebras over non-modular fields which hold for any infinite field. We first have

THEOREM 4. Let $D$ be a normal division algebra of degree $n$ over $F$, and let $Z$ be equivalent to any sub-field of $D$ of degree $n$. Then $D \times Z=D_{Z}$ is a total matric algebra.

Wedderburn's proof* of this theorem holds for an arbitrary field. As an immediate consequence of Theorem 2 we have

TheOREM 5. There exist separable splitting fields of $D$ of degree $n$.

We of course say that $Z$ is a splitting field of $D$ if $D_{Z}$ is a total matric algebra.

We also have Wedderburn's theorems:

Theorem $\dagger$ 6. Let $A$ be a normal simple algebra of degree $n^{2}$ over $F$. Then $A=M \times D \sim D$, where $M$ is a total matric algebra and $D$ is a normal division algebra whose degree is the index of $A$. Moreover $D$ and $M$ are uniquely determined apart from an interior automorphism of $A$.

THEOREM $\ddagger$ 7. Let $B$ be a normal simple algebra over $F$ contained in any algebra $A$ over $F$ with the same modulus as $B$. Then $A=B \times C$ where $C$ also has the same modulus as $A$.

- For Theorems 10, 12, see Wedderburn's paper in these Transactions, vol. 22 (1921), pp. 129135. The proof of Theorem 4 appears on p. 133 and the footnote to p. 134.

† Cf. L. E. Dickson's Algebren, p. 120.

$\ddagger$ Proceedings of the Edinburgh Mathematical Society, vol. 25 (1906-07), pp. 1-3. 
The proofs given by Wedderburn of the above Theorems 6,7 also hold in view of Theorem 5. They may also be applied, as in the non-modular case, to give my

Index Reduction Theorem.* Let $D$ be a normal division algebra of degree (index) $n$ over any infinite field $F, Z$ an algebraic field of degree $r$ over $F$. Then the index of $D_{z}$ over $Z$ is

$$
n^{\prime}=n / s,
$$

where the index reduction factor $s$ divides $r$.

As a consequence we have the whole Brauer exponent theory as well as my

THEOREM $\dagger$ 8. Let $D$ be a normal division algebra of degree $n$ over any infinite field $F, p$ a prime divisor of $n$. Then there exists a field $Z$ of degree $r$ over $F$ such that

$$
D=M \times B \sim B
$$

(M total matric),

where $B$ is a cyclic division algebra of degree $p$ over its centrum $Z$.

THEOREM $\ddagger$. Let $Z_{0}$ be in $D$ so that the degree $r$ of the field $Z_{0}$ divides $n$ and let $Z$ be equivalent to $Z_{0}$

$$
D_{\mathrm{z}}=M \times B,
$$

as in the Index Reduction Theorem. Then the algebra $B_{0}$ over $Z_{0}$ of all quantities of $D$ commutative with every quantity of $Z_{0}$ is equivalent to $B$ over $Z$.

We may indeed say that almost all of the recent general theory on normal division algebras holds when $F$ is any infinite field. The determination theorems on algebras of degree $2,3,4$ do not hold however. We shall give here a determination in the cases $n=2,3$, and, in a later American Journal paper, the case $n=4$. We shall require

ThEOREM 10. Let $D$ be a normal division algebra of degree $n$ over $F$, and let $x$ in $D$ have $\phi(\omega)=0$ of degree $\nu$ as its minimum equation. Then

$$
\phi(\omega) \equiv\left(\omega-x_{v}\right)\left(\omega-x_{v-1}\right) \cdots\left(\omega-x_{2}\right)(\omega-x),
$$

where the $\nu$ factors may be permuted cyclically.

THEOREM $\$$ 11. Every root $y$ in $D$ of $\phi(\omega)=0$ is a transform $t x t^{-1}=y$ of $x$ by t in $D$.

* On direct products, these Transactions, vol. 33 (1931), pp. 690-711.

$\dagger$ For probably the best proof of Theorem 8 see (1), (2) on p. 725 of the joint paper by H. Hasse and myself in these Transactions, vol. 34 (1932), pp. 722-726.

$\ddagger$ On normal simple algebras, these Transactions, vol. 34 (1932), pp. 620-625.

8 Cf. Annals of Mathematics, vol. 30 (1929), pp. 322-338, Theorem 12. 
THEOREM 12. Let $f(\omega) \equiv g(\omega) \cdot h(\omega)$ where $f, g$, h have coefficients in $D$ and $\omega$ is a scalar variable. Then if $\omega-x$ is a right divisor of $f(\omega), h(\omega) \equiv q(\omega)(\omega-x)+R$ where $R \neq 0$ is in $D$, then $\omega-R x R^{-1}$ is a right divisor of $g(\omega)$.

4. Algebras over perfect fields. We may now prove

THEOREM 13. Let $D$ be a normal division algebra of degree $n$ over a perfect modular field $F$ of characteristic $p$. Then $n$ is not divisible by $p$.

For by Theorem 8 , if $n$ is divisible by $p$ then there exists an extension $Z$ of finite degree over $F$, such that $D \times Z=M \times B$ where $B$ is a cyclic division algebra of degree $p$ over $F$. But it is known* that then $Z$ is perfect. Moreover $B=(X, S, \gamma)$ where $X$ is cyclic of degree $p$ over $Z$ and with generating automorphism $S, \gamma$ in $Z$ is not the norm $N(f)$ of any $f$ in $X$. But $Z$ is perfect, $\gamma=\delta^{p}=N(\delta)$, a contradiction.

5. Algebras of degree two. Let $D$ be a normal division algebra of degree two over an infinite field $F$ of characteristic two. By Theorem 2, algebra $D$ contains a separable quadratic field $F(x), x^{2}=\lambda x+\mu$ where $\lambda \neq 0, \mu \neq 0$ are in $F$. We let $i=\lambda^{-1} x$ so that $i^{2}=\lambda^{-2}(\lambda x+\mu)=i+\alpha$ where $\alpha=\mu \lambda^{-2} \neq 0$ is in $F$. The equation $\omega^{2}=\omega+\alpha$ is cyclic and in fact has the roots $i, i+1$. By Theorem 12 there exists a quantity $j$ in $D$ such that $j i=(i+1) j$. But then $j^{2} i=i j^{2}$. Since $F(i)$ is a maximal sub-field of $A$, the quantity $j^{2}$ is in $F(i)$. But $F\left(j^{2}\right)<F(i)$ since $j j^{2}=j^{2} j$, but $j i \neq i j$. Hence $j^{2}=\gamma$ in $F$ and we have proved

THEOREM 14. Every normal division algebra $D$ of degree two over $F$ of characteristic 2 is a cyclic algebra

$$
\begin{gathered}
(1, i, j, i j), \quad i^{2}=i+\alpha, \\
i i=(i+1) j, \quad j^{2}=\gamma,
\end{gathered}
$$

with $\alpha$ and $\gamma$ in $F$.

6. Algebras of degree three. We now let three be the degree of $D$ and the characteristic of $F$. By Theorem 2 there exists a separable cubic sub-field $F(u)$ of $F$ such that $u$ has

$$
\phi(\omega) \equiv \omega^{3}+\alpha \omega^{2}+\beta \omega+\gamma=0,
$$

with $\alpha \neq 0$ by Theorem 3. By Theorem 10 we have

$$
\phi(\omega) \equiv\left(\omega-u_{3}\right)\left(\omega-u_{2}\right)\left(\omega-u_{1}\right)
$$

where $u=u_{1}, u_{2}, u_{3}$ are evidently distinct and $u_{2}, u_{3}$ are transforms of $u$ by

* Cf. E. Steinitz, Algebraische Theorie der Körper, p. 55. 
quantities of $F$. If

$$
x=u_{2} u_{1}-u_{1} u_{2}
$$

is zero then evidently $\phi(\omega)$ is a cyclic equation, $D$ is a cyclic algebra. For $u_{2} u_{1}=u_{1} u_{2}$ implies that $u_{2}$ is in $F\left(u_{1}\right)$.

Hence let $x \neq 0$. By Wedderburn's proof for the case where the characteristic of $F$ is not three, we have

$$
x u_{1}=u_{2} x, \quad x u_{2}=u_{3} x, \quad x u_{3}=u_{1} x,
$$

so that $x^{3} u_{1}=u_{1} x^{3}$ and $x^{3}$ is in $F$. Let then $x^{3}=\delta$ in $F$.

The minimum equation of $x$ with respect to $F$ is

$$
\psi(\omega) \equiv \omega^{3}-\delta \equiv(\omega-x)^{3}=0,
$$

so that $F(x)$ is inseparable and Wedderburn's proof breaks down. But let $v=u_{1} x-x u_{1}=\left(u_{1}-u_{2}\right) x \neq 0$. Write $x=x_{1}$. Then $x_{1} \neq u_{1} x_{1} u_{1}^{-1}$ since $\left(x_{1}-u x_{1} u_{1}^{-1}\right) u_{1}$ $=x u_{1}-u_{1} x=-v \neq 0$. Hence $\omega-u_{1} x_{1} u_{1}^{-1}$ is a right divisor of $\psi(\omega)$ but not of $\omega-x$, and, by Theorem 12 , with $R=u_{1} x_{1} u_{1}^{-1}-x_{1}=v u_{1}^{-1}$ we have $\omega-v x_{1} v^{-1}$ a right divisor of $\left(\omega-x_{1}\right)^{2}$. We have obtained

$$
\left(\omega-x_{1}\right)^{2} \equiv\left(\omega^{2}-2 x_{1} \omega+x_{1}^{2}\right) \equiv\left(\omega-x_{3}\right)\left(\omega-x_{2}\right), \quad x_{2}=v x_{1} v^{-1} .
$$

Now

$$
x_{2}=v x_{1} v^{-1}=\left(u_{1}-u_{2}\right) x_{1}^{2} x_{1}^{-1}\left(u_{1}-u_{2}\right)^{-1}=\left(u_{1}-u_{2}\right) x_{1}\left(u_{1}-u_{2}\right)^{-1} .
$$

But

$$
x_{1}\left(u_{1}-u_{2}\right)=\left(u_{2}-u_{3}\right) x_{1},\left(u_{2}-u_{3}\right)^{-1} x_{1}=x_{1}\left(u_{1}-u_{2}\right)^{-1}
$$

and

$$
x_{2}=\left(u_{1}-u_{2}\right)\left(u_{2}-u_{3}\right)^{-1} x_{1} .
$$

If $x_{2}=x_{1}$ then $u_{1}-u_{2}=u_{2}-u_{3}$. But $3 u_{2}=0, u_{1}-2 u_{2}+u_{3}=u_{1}+u_{2}+u_{3}=0=\alpha$, a contradiction. Hence $x_{2} \neq x_{1}$. Also $x_{3}+x_{2}+x_{1}=0, x_{3}+x_{2}=2 x_{1}, x_{3}-x_{1}$ $=x_{1}-x_{2} \neq 0, x_{3}-x_{2}=2\left(x_{1}-x_{2}\right) \neq 0$. Thus $x_{3}, x_{2}, x_{1}$ are all distinct and we have obtained a factorization in $D$ of $\psi(\omega)$ into distinct factors in spite of the fact that $\psi(\omega)=0$ is inseparable.

Moreover $\left(\omega-x_{1}\right)^{3} \equiv\left(\omega-x_{2}\right)^{3} \equiv\left(\omega-x_{3}\right)^{3} \equiv\left(\omega-x_{1}\right)\left(\omega-x_{3}\right) \quad\left(\omega-x_{2}\right)$, so that $\left(\omega-x_{2}\right)^{2}-\left(\omega-x_{1}\right)\left(\omega-x_{3}\right)$ and $x_{1} x_{3}=x_{2}{ }^{2}$.

If $x_{2} x_{1}-x_{1} x_{2}=0$, then $x_{2} \neq x_{1}$ is in $F\left(x_{1}\right),\left(x_{2}-x_{1}\right)^{3}=x_{2}^{8}-x_{1}^{8}=0$, a contradiction. Hence $y=\dot{x}_{2} x_{1}-x_{1} x_{2} \neq 0$. By the Wedderburn proof*

$$
y x_{1}=x_{2} y, y x_{2}=x_{3} y, y x_{3}=x_{1} y, y^{3}=\epsilon \text { in } F .
$$

* These Transactions (loc. cit.), 1921. 
We let $z_{1}=x_{1} y, z_{2}=y z_{1} y^{-1}=y x_{1} y y^{-1}=y x_{1}$, a transform of $z_{1}$ by $y$. Also $y x_{1} \neq x_{1} y$ so that $z_{2} \neq z_{1}$. Thus $z_{2} z_{1}-z_{1} z_{2}=y x_{1}^{2} y-x_{1} y^{2} x_{1}=\left(x_{2}^{2}-x_{3} x_{1}\right) y^{2}=0$. Hence $z_{2}$ is commutative with $z_{1}, z_{2}$ is in $F\left(z_{1}\right), z_{2} \neq z_{1}$ and $F\left(z_{1}\right)$ is cyclic. We have proved THEOREM 15. Every normal division algebra of degree three over any infinite field $F$ is cyclic.

The Institute for Advanced Study, Princeton, N. J. 